PTA/08-053

\title{
Four-point function in Super Liouville Gravity
}

\author{
A. Belavin \\ L. D. Landau Institute for Theoretical Physics RAS \\ 142432 Chernogolovka, Russia \\ and \\ V. Belavin ${ }^{1}$ \\ Laboratoire de Physique Théorique et Astroparticules \\ Université Montpellier II \\ Pl. E. Bataillon, 34095 Montpellier, France
}

\begin{abstract}
We consider the 2D super Liouville gravity coupled to the minimal superconformal theory. We analyze the physical states in the theory and give the general form of the $n$-point correlation numbers on the sphere in terms of integrals over the moduli space. The threepoint correlation numbers are presented explicitly. For the four-point correlators, we show that the integral over the moduli space reduces to the boundary terms if one of the fields is degenerate. It turns out that special logarithmic fields are relevant for evaluating these boundary terms. We discuss the construction of these fields and study their operator product expansions. This analysis allows evaluating the four-point correlation numbers. The derivation is analogous to the one in the bosonic case and is based on the recently derived higher equations of motion of the super Liouville field theory.
\end{abstract}

\section{Introduction}

Super Liouville gravity (SLG) [1] is the two-dimensional quantum gravity whose action is induced by a super conformal matter. This induced action is universal (i.e., its form is independent of the concrete choice of the conformal matter) and is known as the super Liouville action. In the framework of the David and Distler-Kawai approach (DDK) [2,3], SLG is presented as a tensor product of a matter theory (SCFT), super Liouville (SLFT) system,

\footnotetext{
${ }^{1}$ Institute of Theoretical and Experimental Physics, B. Cheremushkinskaya 25, 117259 Moscow, Russia.
} 
and super ghost (SG) system. The SG system also has super conformal symmetry and appears as a result of the gauge-fixing problem in SLG (see, e.g., [4] for details). Schematically, the SLG action is

$$
A_{\mathrm{SLG}}=A_{\mathrm{SCFT}}+A_{\mathrm{SL}}+A_{\mathrm{SG}} \text {. }
$$

All three theories constituting SLG are completely solvable by the standard bootstrap technique [5] (at least in principle) because of the infinite symmetry given by the superconformal algebra

$$
\begin{aligned}
{\left[L_{n}, L_{m}\right] } & =(n-m) L_{n+m}+\frac{c}{8}\left(n^{3}-n\right) \delta_{n ;-m}, \\
\left\{G_{r}, G_{s}\right\} & =2 L_{r+s}+\frac{c}{2}\left(r^{2}-\frac{1}{4}\right) \delta_{r ;-s}, \\
{\left[L_{n}, G_{r}\right] } & =\left(\frac{1}{2} n-r\right) G_{n+r},
\end{aligned}
$$

where $c$ is the central charge parameter and the indices $r$ and $s$ are integers for the Ramond (R) sector and half-integers for the Neveu-Schwarz (NS) sector. We restrict ourself to considering only the NS sector here. Whenever it cannot cause confusion, we omit sector indices for the superconformal generators (i.e., indices indicating which sector-Liouville, matter, or ghost - is under consideration).

The interaction between the three components of SLG in the DDK approach is via the relation for the central charge parameters

$$
c_{\mathrm{SCFT}}+c_{\mathrm{SL}}+c_{\mathrm{SG}}=0
$$

and also due to the construction of the physical fields and the integration over the moduli space in constructing the correlation numbers (see below).

This paper is organized as follows. In the next three sections, we briefly recall some relevant aspects concerning all three ingredients of SLG. Section 5 is devoted to the preliminary analysis of the physical states in SLG. We use the material in the preceding sections to construct the basic types of the physical fields in the standard framework of BRST quantization. We also introduce the special discrete series of physical states, known as "ground ring elements" and discuss the relation between the logarithmic counterparts of the ground ring elements and the basic physical fields. This is our first main result. In the next section, we discuss the operator products of the ground ring elements with the basic physical fields. In Sec. 7 , we derive the general form of the $n$-point correlation number on the sphere in SLG. This expression contains the integration over the moduli space. The simplest case of three points, when the moduli space is trivial, is given explicitly. For the four-point correlators, we show that in special cases the integration over moduli reduces to the boundary terms, which are defined by the operator product expansions (OPEs) of the "logarithmic" fields. In Sec. 8, we calculate all necessary operator products explicitly and evaluate the boundary terms. Finally, this consideration leads to an explicit expression for the four-point correlation number of one degenerate and three generic fields, which is presented in Sec. 9. This is our second main result. Some calculations omitted in the main text are presented in the appendices. 


\section{Super Liouville field theory}

The super Liouville field theory is a super conformal field theory with the central charge

$$
c=1+2 Q^{2}
$$

where the "background charge" $Q$ is parameterized in terms of the basic "quantum" parameter $b$ as $Q=b^{-1}+b$. The classical (as well as the quantum) SLFT has been introduced and studied in [6-9] shortly after it appeared in the string context in [1]. Below, we present the main facts concerning NS sector of SLFT (see [10-13] and the references therein for more details and recent developments). The NS fields belong to the highest-weight representations of the superconformal algebra. The basic fields are the scalar primary fields $V_{a}(x)$ corresponding to the highest-weight vectors

$$
\begin{aligned}
& L_{n} V_{a}=0, \quad \bar{L}_{n} V_{a}=0, \quad \text { for } n>0, \\
& G_{k} V_{a}=0, \quad \bar{G}_{k} V_{a}=0, \quad \text { for } k>0, \\
& L_{0} V_{a}=\bar{L}_{0} V_{a}=\Delta_{a} V_{a},
\end{aligned}
$$

where

$$
\Delta_{a}=\frac{a(Q-a)}{2}
$$

and $a$ is a (complex) continuous parameter. We also use another parameter $\lambda=Q / 2-a$. The representations are singular at certain special values of the parameters. This happens [14] at $\lambda=\lambda_{m, n}$, where $(m, n)$ is a pair of positive integers $(m-n \in 2 \mathbb{Z})$ and

$$
\lambda_{m, n}=\frac{m b^{-1}+n b}{2} .
$$

In general, at $a=a_{m, n}$, one singular vector appears at the level $m n / 2$ in the Verma module over $V_{a_{m, n}}=V_{m, n}$. For each pair $(m, n)$, it is convenient to introduce a "singular-vector creation operator" $D_{m, n}$, which is a graded polynomial in $G_{-k}$ and $L_{-k}$ of level $m n / 2$ whose coefficients are functions of the central charge parameter $b^{2}$ such that the singular vector appears when $D_{m, n}$ is applied to $V_{m, n}$. The normalization is unambiguously fixed via the coefficient of the highest-order term $D_{m, n}=G_{-1 / 2}^{m n}+\ldots$ The basic OPE is

$$
V_{a_{1}}(x) V_{a_{2}}(0)=\int \frac{d P}{4 \pi}(x \bar{x})^{\Delta-\Delta_{1}-\Delta_{2}}\left(\mathbb{C}_{a_{1}, a_{2}}^{Q / 2+i P}\left[V_{Q / 2+i P}(0)\right]_{\mathrm{ee}}+\tilde{\mathbb{C}}_{a_{1}, a_{2}}^{Q / 2+i P}\left[V_{Q / 2+i P}(0)\right]_{\mathrm{oo}}\right)
$$

(for brevity here and hereafter, we set $\Delta=\Delta_{Q / 2+i P}$ and $\Delta_{i}=\Delta_{a_{i}}$ ). This OPE is continuous and involves integration over the "momentum" $P$. In $(8),\left[V_{p}\right]$ denotes the contribution of the primary field $V_{p}$ and its superconformal descendants to the OPE (subscript ee stands for the descendants on the integer level and oo - for the descendants on the half-integer level). All other OPEs of two arbitrary local fields can be derived from (8). The basic structure 
constants $\mathbb{C}_{a_{1} a_{2}}^{Q / 2+i P}$ and $\widetilde{\mathbb{C}}_{a_{1}, a_{2}}^{Q / 2+i P}$ in (8) were evaluated using the bootstrap technique in [15-17] and have the explicit form (here $a$ denotes $a_{1}+a_{2}+a_{3}$ )

$$
\begin{aligned}
& \mathbb{C}_{a_{1} a_{2}}^{Q-a_{3}}=\left(\pi \mu \gamma\left(\frac{Q b}{2}\right) b^{1-b^{2}}\right)^{(Q-a) / b} \frac{\Upsilon_{\mathrm{R}}(b) \Upsilon_{\mathrm{NS}}\left(2 a_{1}\right) \Upsilon_{\mathrm{NS}}\left(2 a_{2}\right) \Upsilon_{\mathrm{NS}}\left(2 a_{3}\right)}{2 \Upsilon_{\mathrm{NS}}(a-Q) \Upsilon_{\mathrm{NS}}\left(a_{1+2-3}\right) \Upsilon_{\mathrm{NS}}\left(a_{2+3-1}\right) \Upsilon_{\mathrm{NS}}\left(a_{3+1-2}\right)}, \\
& \tilde{\mathbb{C}}_{a_{1} a_{2}}^{Q-a_{3}}=-\left(\pi \mu \gamma\left(\frac{Q b}{2}\right) b^{1-b^{2}}\right)^{(Q-a) / b} \frac{i \Upsilon_{\mathrm{R}}(b) \Upsilon_{\mathrm{NS}}\left(2 a_{1}\right) \Upsilon_{\mathrm{NS}}\left(2 a_{2}\right) \Upsilon_{\mathrm{NS}}\left(2 a_{3}\right)}{\Upsilon_{\mathrm{R}}(a-Q) \Upsilon_{\mathrm{R}}\left(a_{1+2-3}\right) \Upsilon_{\mathrm{R}}\left(a_{2+3-1}\right) \Upsilon_{\mathrm{R}}\left(a_{3+1-2}\right)},
\end{aligned}
$$

where we use the convenient notation in [17] for the special functions

$$
\begin{aligned}
\Upsilon_{\mathrm{NS}}(x) & =\Upsilon_{b}\left(\frac{x}{2}\right) \Upsilon_{b}\left(\frac{x+Q}{2}\right) \\
\Upsilon_{\mathrm{R}}(x) & =\Upsilon_{b}\left(\frac{x+b}{2}\right) \Upsilon_{b}\left(\frac{x+b^{-1}}{2}\right)
\end{aligned}
$$

expressed in terms of the "upsilon" function $\Upsilon_{b}$, which is standard in the Liouville field theory (see $[18, ?])$. Structure constants $(9)$ correspond to the normalization of the primary fields

$$
\left\langle V_{a} V_{Q-a}\right\rangle_{\mathrm{SLFT}}=(x \bar{x})^{-2 \Delta_{a}} .
$$

An important result concerning SLFT is the higher equations of motion. Following [20], we define the set of "logarithmic degenerate fields" in the NS sector

$$
V_{m, n}^{\prime}=\left.V_{a}^{\prime}\right|_{a=a_{m, n}}, \quad m-n \in 2 \mathbb{Z},
$$

where the general logarithmic fields $V_{a}^{\prime}=\partial V_{a} / \partial a$ are the derivatives with respect to $a$ of the corresponding primary fields. It turns out that while $V_{m, n}^{\prime}$ are logarithmic fields (as well as general $V_{a}^{\prime}$ ), the fields

$$
\bar{D}_{m, n} D_{m, n} V_{m, n}^{\prime}
$$

have the properties of primary fields and should be identified with the exponential primary fields $V_{m,-n}$. More precisely, we have the relations

$$
\bar{D}_{m, n} D_{m, n} V_{m, n}^{\prime}=B_{m, n} V_{m,-n}
$$

known as the higher equations of motion. Here, the exponential primaries $V_{m,-n}$ have the dimensions $\Delta_{m, n}+m n / 2$ and the coefficients

$$
B_{m, n}=2^{m n} i^{m n-2[m n / 2]} b^{n-m+1}[\pi \mu \gamma(b Q / 2)]^{n} \gamma\left(\frac{m-n b^{2}}{2}\right) \prod_{(k, l) \in\langle m, n\rangle_{\mathrm{NS}}} \lambda_{k, l}
$$

where the set $\langle m, n\rangle_{\mathrm{NS}}$ is the set of integer pairs

$$
\{(k, l) \in\{k \mid 1-m \leq k \leq m-1\},\{l \mid 1-n \leq l \leq n-1\} \mid k-l \in 2 \mathbb{Z}\} \backslash\{(0,0)\} .
$$




\section{$3 \quad$ Generalized super minimal models}

We consider the special type of SLG where the so-called generalized super minimal models (GSMM) are in the matter sector of the theory. We call the corresponding induced Liouville gravity the minimal super Liouville gravity. In the GSMM, there are no special restrictions on the central charge, which can take an arbitrary value in principle, in contrast to the case of ordinary minimal models. It is instructive to parameterize the central charge via the same basic parameter $b$ as for SLFT,

$$
c=1-2\left(b^{-1}-b\right)^{2} .
$$

In this parameterization, condition (3) for the total central charge is satisfied automatically. The "canonical" super minimal models appear for the special choice of the parameter $b$ such that $b^{2}$ is a rational number. Otherwise, the algebra of the degenerate primary fields no longer closes within any finite subset; instead, the whole set $\left\{\Phi_{m, n}\right\}$ with any pair $(m, n)$ of natural numbers forms a closed algebra. Moreover, we enlarge the space of local fields by including local fields with dimensions different from the Kac values. Hence, the spectrum of dimensions is continuous in GSMM. We introduce the continuous parameter $\alpha$ to parameterize a continuous family $\left\{\Phi_{\alpha}\right\}$ of primary fields with the dimensions

$$
\Delta_{\alpha}^{(\mathrm{M})}=\frac{\alpha(\alpha-q)}{2}
$$

where

$$
q=b^{-1}-b .
$$

We also always use the "canonical" CFT normalization of the primary fields $\Phi_{\alpha}$ via the two-point functions

$$
\left\langle\Phi_{\alpha} \Phi_{\alpha}\right\rangle_{\mathrm{GSMM}}=(x \bar{x})^{-2 \Delta_{\alpha}} .
$$

The degenerate fields $\Phi_{m, n}$ have the dimensions

$$
\Delta_{m, n}^{(\mathrm{M})}=-q^{2} / 8+\lambda_{m,-n}^{2} / 2
$$

They correspond to either $\alpha=\alpha_{m, n}$ or $\alpha=q-\alpha_{m, n}$ with

$$
\alpha_{m, n}=q / 2+\lambda_{-m, n}
$$

It can be seen that the construction of GSMM is formally similar to that of SLFT and differs by the change $b \rightarrow i b$ and $\alpha \rightarrow-i a$ and also by the normalization conditions for the primary fields, Eq. (11) for SLFT and Eq. (20) for GSMM.

\section{Super ghosts}

In this section, we collect some results concerning SG (see, e.g., [21, ?,23] for details). The SG appear as a result of gauge fixing in the Polyakov approach to SLG and are described by the free super conformal field theory with the central charge $c_{\mathrm{SG}}=-10$. 
The fermionic part of the SG system involves two anticommuting fields $(b, c)$ of spins $(2,-1)$ with the action

$$
A_{\mathrm{bc}}=\frac{1}{2 \pi} \int d^{2} z(b \bar{\partial} c+\bar{b} \partial \bar{c})
$$

The operator products are readily found, with appropriate attention to the order of the anticommuting variables,

$$
b(z) c(0) \sim \frac{1}{z}, \quad b(z) b(0) \sim O(z), \quad c(z) c(0) \sim O(z) .
$$

As usual we focus on the holomorphic part. The fields have the Laurent expansions

$$
b(z)=\sum_{m=-\infty}^{\infty} \frac{b_{m}}{z^{m+2}}, \quad c(z)=\sum_{m=-\infty}^{\infty} \frac{c_{m}}{z^{m-1}},
$$

which yield the anticommutators

$$
\left\{b_{m}, c_{n}\right\}=\delta_{m+n, 0}, \quad\left\{b_{m}, b_{n}\right\}=0, \quad\left\{c_{m}, c_{n}\right\}=0 .
$$

There are two natural ground states, both are annihilated by $b_{m}$ and $c_{m}$ for $m>0$. In addition, the first one is annihilated by $b_{0}$, and the second one is annihilated by $c_{0}$. For future purposes, we choose the vacuum annihilated by $b_{0}$ :

$$
\begin{aligned}
& b_{m}|v\rangle_{b c}=0, \quad m \geq 0, \\
& c_{m}|v\rangle_{b c}=0, \quad m \geq 1 .
\end{aligned}
$$

It can be verified that the vacuum $|v\rangle_{b c}$ corresponds to the field $c(x)$ with the dimension -1 . The unit operator, which is relevant later, corresponds to the state

$$
|1\rangle_{b c}=b_{-1}|v\rangle_{b c} .
$$

All other representations of (26) are equivalent to the one described above.

The bosonic part of the SG involves two bosonic fields $(\beta, \gamma)$ of spins $(3 / 2,-1 / 2)$ that are superpartners of the respective fermionic ghosts $(b, c)$. The action has the form

$$
A_{\beta \gamma}=\frac{1}{2 \pi} \int d^{2} z(\beta \bar{\partial} \gamma+\bar{\beta} \partial \bar{\gamma})
$$

Because the statistics changes, some signs in the operator products change:

$$
\beta(z) \gamma(0) \sim-\frac{1}{z}, \quad \gamma(z) \beta(0) \sim \frac{1}{z}, \quad \beta(z) \beta(0) \sim O(1), \quad \gamma(z) \gamma(0) \sim O(1) .
$$

The Hilbert space of the $(\beta, \gamma)$-system is constructed by expanding

$$
\beta(z)=\sum_{m=-\infty}^{\infty} \frac{\beta_{m}}{z^{m+3 / 2}}, \quad \gamma(z)=\sum_{m=-\infty}^{\infty} \frac{\gamma_{m}}{z^{m-1 / 2}}
$$


where index $m$ is integer for the $\mathrm{R}$ sector and half-integer for the NS sector and the coefficients satisfy the canonical commutation relations

$$
\left[\gamma_{m}, \beta_{n}\right]=\delta_{m+n, 0}, \quad\left[\beta_{m}, \beta_{n}\right]=0, \quad\left[\gamma_{m}, \gamma_{n}\right]=0
$$

The state-operator map is now trickier. In contrast to the fermionic case, there now exist discreet series of vacuums $\left\{|q\rangle_{\beta \gamma}\right\}$ with $q$ either integer or half-integer,

$$
\begin{array}{ll}
\beta_{m}|q\rangle_{\beta \gamma}=0, & m \geq-q-1 / 2, \\
\gamma_{m}|q\rangle_{\beta \gamma}=0, & m \geq q+3 / 2 .
\end{array}
$$

Each $q$-vacuum defines an inequivalent representation of (32), sometimes called a "picture." States in the Hilbert space are in a one-to-one correspondence with local field operators, and the complete space of local fields is therefore the direct sum of all highest-weight representations with the highest vectors $|q\rangle_{\beta \gamma}$. We define the two local operators that are most important for the subsequent developments. It can be verified that the vacuum $|q=0\rangle_{\beta \gamma}$ has the dimension 0 and corresponds to the unit operator. The local operator corresponding to the vacuum $|q=-1\rangle_{\beta \gamma}$ is particularly useful. It turns out that this vacuum corresponds to the formal operator $\delta(\gamma(0))$ of dimension 1/2. Indeed, taking the general properties of the Dirac $\delta$-function and OPE (32) (see Appendix A) into account, we can straightforwardly derive the operator product relations

$$
\gamma(z) \delta(\gamma(0)) \sim z \partial \gamma(0) \delta(\gamma(0)), \quad \beta(z) \delta(\gamma(0)) \sim-\frac{1}{z} \delta(\gamma(0))
$$

and similar equations with $\beta$ and $\gamma$ interchanged. Using these expansions we can easily verify that $\delta(\gamma(0))$ satisfies $(33)$ for $q=-1$.

The anomaly in the conservation of the ghost currents $J^{b c}(u)=-: b(u) c(u)$ : and $J^{\beta \gamma}(u)=$ $-: \beta(u) \gamma(u)$ : leads to the important requirement for the correlation functions. In particular, on the sphere, any correlation function in SLG including ghost observables built of $\beta, \gamma$, $\delta(\beta(0)), \delta(\gamma(0))$, and their descendants should satisfy the ghost-number balance

$$
\begin{aligned}
& N_{c}-N_{b}=3 \\
& \left(N_{\delta(\gamma)}-N_{\delta(\beta)}\right)+\left(N_{\beta}-N_{\gamma}\right)=2 .
\end{aligned}
$$

Although this relation is known (see, e.g., [21]), we rederive this result in Appendix A. Finally, the superconformal generators in the ghost sector are

$$
\begin{aligned}
L_{m}^{g} & =\sum_{n}(m+n): b_{m-n} c_{n}:+\sum_{k}\left(\frac{m}{2}+k\right): \beta_{m-k} \gamma_{k}:-\frac{1}{2} \delta_{m, 0}, \\
G_{k}^{g} & =-\sum_{n}\left[\left(k+\frac{n}{2}\right) \beta_{k-n} c_{n}+2 b_{n} \gamma_{k-n}\right],
\end{aligned}
$$


where the normal ordering is defined with respect to the ground states $|v\rangle_{b c}$ and $|q=-1\rangle_{\beta \gamma}$. In the practical calculations, the computation relations of the ghost generators to the superconformal generator $L_{n}$,

$$
\begin{aligned}
& {\left[L_{n}, b_{m}\right]=(n-m) b_{n+m}} \\
& {\left[L_{n}, c_{m}\right]=-(2 n+m) c_{n+m},} \\
& {\left[L_{n}, \beta_{k}\right]=\left(\frac{n}{2}-k\right) \beta_{n+k},} \\
& {\left[L_{n}, \gamma_{k}\right]=-\left(\frac{3 n}{2}+k\right) \gamma_{n+k}}
\end{aligned}
$$

and to the generator $G_{k}$,

$$
\begin{aligned}
& {\left[G_{k}, b_{n}\right]=\left(\frac{n}{2}-k\right) \beta_{k+n},} \\
& {\left[G_{k}, c_{n}\right]=-2 \gamma_{k+n},} \\
& {\left[G_{k}, \beta_{r}\right]=-2 b_{k+r},} \\
& {\left[G_{k}, \gamma_{r}\right]=\left(\frac{3 k}{2}+\frac{r}{2}\right) c_{k+r},}
\end{aligned}
$$

are relevant. In the next section, we use the facts described above to construct the basic physical states in SLG.

\section{Physical states in SLG}

The vanishing of the total central charge (3) is not sufficient to ensure the Weyl invariance of SLG. Inserting the physical fields in the functional integral should also not spoil Weyl invariance. This leads to a certain restriction on the structure of the physical fields. The standard mathematical tool for treating such problems is cohomology theory. The physical fields form a space of cohomology classes with respect to the nilpotent BRST charge $Q$. In modes, $Q$-operator can be derived via the commutation relations

$$
\begin{aligned}
& \left\{b_{n}, Q\right\}=L_{n}, \\
& {\left[\beta_{r}, Q\right]=G_{r},}
\end{aligned}
$$

Here and below $L_{n}$ and $G_{r}$ are the total superconformal generators, defined by the sum of the corresponding generators in all three sectors (matter, Liouville, and ghost),

$$
\begin{aligned}
& L_{n}=L_{n}^{\mathrm{M}}+L_{n}^{\mathrm{L}}+L_{n}^{\mathrm{g}}, \\
& G_{r}=G_{r}^{\mathrm{M}}+G_{r}^{\mathrm{L}}+G_{r}^{\mathrm{g}} .
\end{aligned}
$$

We also will use the notations $L_{n}^{\mathrm{M}+\mathrm{L}}=L_{n}^{\mathrm{M}}+L_{n}^{\mathrm{L}}$ and $G_{r}^{\mathrm{M}+\mathrm{L}}=G_{r}^{\mathrm{M}}+G_{r}^{\mathrm{L}}$. The commutation relations (39) lead to the expansion of the BRST operator

$$
Q=\sum_{m}:\left[L_{m}^{\mathrm{M}+\mathrm{L}}+\frac{1}{2} L_{m}^{\mathrm{g}}\right] c_{-m}:+\sum_{r}:\left[G_{r}^{\mathrm{M}+\mathrm{L}}+\frac{1}{2} G_{r}^{\mathrm{g}}\right] \gamma_{-r}:-\frac{1}{4} c_{0},
$$


where $m$ ranges the integers, $r$ ranges the half-integers, and the normal ordering is the same as in Eq. (36). From this, we can verify the nilpotence whenever the total central charge vanishes. The observable spectrum consists of the BRST invariant local fields,

$$
Q|\Psi\rangle=0,
$$

which not belong to $\operatorname{Im} Q$. Because of (39), the total dimension of any nontrivial physical state is zero. Besides the local fields, for the construction of the physical amplitudes in SLG we introduce also non-local physical fields of the form

$$
\int \bar{b}_{-1} b_{-1} \Psi(z, \bar{z}) d^{2} z,
$$

where $\Psi(z, \bar{z})$ satisfies (42). The gauge invariance of these fields follows directly from (39). We do not completely classify the physical states in SLG for all different pictures here. Our aim here to bring sufficient information into consideration to construct a gravitational $n$ point correlation number for some special choice of the pictures. As it was mentioned above, the ghost number balance (35) should be satisfied on the sphere. This condition can be fulfilled by choosing the fields in such a way that three of them have the ghost factors $c$ and $\bar{c}$ and two fields have the factors $\delta(\gamma)$ and $\delta(\bar{\gamma})$. In particular it means that two of the field belong to the picture $q=-1$ and others belong to the picture $q=0$. We therefore concentrate on the physical states of this kind in what follows. Apparently, the general case is obtained from this special one by applying the picture changing operator [21].

It is natural to start our search of the physical states among those constructed from the primary fields in all three sectors. We call them the "basic" physical states. We introduce the special notation for the vacuum vector in the tensor product of the matter and Liouville sectors

$$
\left|U_{a}\right\rangle=\left|\Phi_{a-b}\right\rangle\left|V_{a}\right\rangle .
$$

The vector $U_{a}$ corresponds to the field of total dimension $(1 / 2,1 / 2)$. We also define the ground state related to the given picture $q$ :

$$
\left|\Omega_{a}\right\rangle_{q}=\left|U_{a}\right\rangle|v\rangle_{b c}|q\rangle_{\beta \gamma} .
$$

It can be verified that

$$
\left|W_{a}\right\rangle=\left|\Omega_{a}\right\rangle_{-1}
$$

satisfies (42). Hence, the physical fields of the first type are

$$
W_{a}(z, \bar{z})=U_{a}(z, \bar{z}) \cdot c(z) \bar{c}(\bar{z}) \cdot \delta(\gamma(z)) \delta(\bar{\gamma}(\bar{z})) .
$$

Similarly, it can be verified that another type of physical states can be defined in the $q=0$ picture

$$
\left|\tilde{W}_{a}\right\rangle=\left(\bar{G}_{-1 / 2}^{\mathrm{M}+\mathrm{L}}+\frac{1}{2} \bar{G}_{-1 / 2}^{\mathrm{g}}\right)\left(G_{-1 / 2}^{\mathrm{M}+\mathrm{L}}+\frac{1}{2} G_{-1 / 2}^{\mathrm{g}}\right)\left|\Omega_{a}\right\rangle_{0} .
$$


In the field language,

$$
\tilde{W}_{a}(z, \bar{z})=\left(\bar{G}_{-1 / 2}^{\mathrm{M}+\mathrm{L}}+\frac{1}{2} \bar{G}_{-1 / 2}^{\mathrm{g}}\right)\left(G_{-1 / 2}^{\mathrm{M}+\mathrm{L}}+\frac{1}{2} G_{-1 / 2}^{\mathrm{g}}\right) U_{a}(z, \bar{z}) \cdot \bar{c}(\bar{z}) c(z) .
$$

To give an idea of the explicit calculations, we prove that $\tilde{W}_{a}$ represents a cohomology class in Appendix B.

The integral version of the physical state (43), corresponding to $\Psi(z, \bar{z})=\tilde{W}_{a}(z, \bar{z})$, is

$$
\int \bar{G}_{-1 / 2} G_{-1 / 2} U_{a}(z, \bar{z}) d^{2} z
$$

An additional "discrete" physical state arises when the representation in the matter sector is degenerate. The importance of the discrete states [24,25], for calculating the correlators in the bosonic LG was recently shown in [29]. It is natural to assume a similar effect in the supersymmetric extension of LG. We start by describing the construction for the discrete states in SLG. The degenerate matter fields $\Phi_{m, n}$, when combined with the degenerate exponentials $V_{m, n}$ of the corresponding SLFT, yield nontrivial BRST invariant operators of ghost number zero,

$$
O_{m, n}(z, \bar{z})=\bar{H}_{m, n} H_{m, n} \Phi_{m, n}(z, \bar{z}) V_{m, n}(z, \bar{z}) .
$$

The operators $H_{m, n}$ are composed of the super Virasoro generators of the level $(m n-1) / 2$ in the matter and Liouville sectors and also of ghost fields. The condition that the field $O_{m, n}$ is closed but nontrivial, i.e., represents a cohomology class, defines the operator $H_{m, n}$ uniquely modulo exact terms. There is an important relation between the discrete states and the physical operators discussed above. We introduce the operator

$$
J_{m, n}(z)=\left(G_{-1 / 2}^{\mathrm{M}+\mathrm{L}}-\frac{1}{2} G_{-1 / 2}^{\mathrm{g}}\right) D_{m, n}^{\mathrm{L}} c(z),
$$

where the action of this operator is simply defined by the left multiplication. Below we omit the dependence on $z$ and $\bar{z}$. Then the basic relation

$$
\bar{Q} Q O_{m, n}=\bar{J}_{m, n} J_{m, n} \Phi_{m, n} V_{m, n}
$$

holds, where we temporarily assume that the singular vector in the Liouville sector is not decoupled. In Appendix $\mathrm{C}$, we prove this relation and also use it to derive the important statement

$$
\bar{Q} Q O_{m, n}^{\prime}=B_{m, n} \tilde{W}_{m,-n}
$$

where we introduce the logarithmic counterparts of the discrete states $O_{m, n}$,

$$
O_{m, n}^{\prime}=\bar{H}_{m, n} H_{m, n} \Phi_{m, n} V_{m, n}^{\prime},
$$

and $B_{m, n}$ is just the coefficients in the higher equations of motion of SLFT (14). We note that in the space of states enlarged by the logarithmic fields, the field $\tilde{W}_{m, n}$ becomes trivial. ${ }^{2}$

\footnotetext{
${ }^{2}$ Naively, this would lead to trivial results for the correlation functions with any other physical fields. But we see below that because of the transformation properties of the BRST current, this does not happen.
} 
Another useful consequence of (54) can be obtained by applying $\bar{b}_{-1} b_{-1}$,

$$
\bar{G}_{-1 / 2} G_{-1 / 2} U_{m,-n}=B_{m, n}^{-1}\left(\bar{\partial}-\bar{Q} \bar{b}_{-1}\right)\left(\partial-Q b_{-1}\right) O_{m, n}^{\prime}
$$

or

$$
\bar{G}_{-1 / 2} G_{-1 / 2} U_{m,-n}=B_{m, n}^{-1} \bar{\partial} \partial O_{m, n}^{\prime} \bmod Q
$$

This means that the integrand in (50) is the full derivative modulo BRST exact terms.

Apparently [26], the particular cases described above correspond to the following general structure of the space of physical states in SLG. In each picture, there is only one cohomology if the representation in the matter sector is not degenerate. In the degenerate case, two additional physical fields arise. For example, in the picture $q=0$, there are fields $\tilde{W}_{m, n}$ and $O_{m, n}$ and the third type of cohomology of the ghost number $N_{g}=2$, which we do not discuss here.

\section{Ground ring operator products}

The discrete states $O_{m, n}$ act modulo exact forms in the space of physical states of the given type (in our case either $W_{a}$ or $\tilde{W}_{a}$ ) because their action does not change the ghost number and all nontrivial classes in the given pictures are generically exhausted by these composite fields with different $a$. Moreover, because of the fusion restrictions of the degenerate fields $\Phi_{m, n}$ and $V_{m, n}$ in the OPE, the general structure of the operator products is

$$
\begin{aligned}
& O_{m, n} W(a)=\sum_{\{r, s\} \in(m, n)} A_{r, s}^{(m, n)} W\left(a+\lambda_{r, s}\right), \\
& O_{m, n} \tilde{W}(a)=\sum_{\{r, s\} \in(m, n)} \tilde{A}_{r, s}^{(m, n)} \tilde{W}\left(a+\lambda_{r, s}\right),
\end{aligned}
$$

where the set of integers $(m, n)$ is defined by the restrictions on the OPE of the degenerate fields in both the matter and the Liouville sectors. To evaluate the numerical coefficients $A_{r, s}^{(m, n)}$ and $\tilde{A}_{r, s}^{(m, n)}$, it is useful to calculate explicitly in the simplest nontrivial case $(m, n)=$ $(1,3)$. In this case, $a_{1,3}=-b$. Equation (53) defines $H_{m n}$ explicitly. For $H_{1,3}$, we find

$$
H_{1,3}=L_{-1}^{\mathrm{M}}-L_{-1}^{\mathrm{L}}-G_{-1 / 2}^{\mathrm{M}} G_{-1 / 2}^{\mathrm{L}}+b^{2} \beta_{-3 / 2} \gamma_{1 / 2}+2 b^{2} b_{-2} c_{1}-b^{2}\left(G_{-1 / 2}^{\mathrm{M}}+G_{-1 / 2}^{\mathrm{L}}\right) \beta_{-3 / 2} c_{1}
$$

and the corresponding field

$$
\begin{aligned}
O_{13}(x)= & \Phi_{13}^{\prime}(x) V_{13}(x)-\Phi_{13}(x) V_{13}^{\prime}(x)-\Psi_{13}(x) \Lambda_{13}(x)+\left[b^{2}: \beta(x) \gamma(x):\right. \\
& \left.+2 b^{2}: b(x) c(x):\right] \Phi_{13}(x) V_{13}(x)-b^{2} \beta(x) c(x) \Psi_{13}(x) V_{13}(z) \\
& -b^{2} \beta(x) c(x) \Phi_{13}(x) \Lambda_{13}(z) .
\end{aligned}
$$


Here, we introduce the special notation for the top components of the primary supermultiplets in the Liouville ${ }^{3}$ and matter sectors

$$
\begin{aligned}
& \Lambda_{a}=\bar{G}_{-1 / 2}^{\mathrm{L}} G_{1 / 2}^{\mathrm{L}} V_{a}, \\
& \Psi_{a}=\bar{G}_{-1 / 2}^{\mathrm{M}} G_{1 / 2}^{\mathrm{M}} \Phi_{a} .
\end{aligned}
$$

We first consider the operator product $O_{13}(x) W_{a}(0)$. The special OPE that we need in this case are those of the degenerate field $V_{13}$ and $\Lambda_{13}$ in the Liouville sector (see [10,11] for details)

$$
\begin{aligned}
V_{1,3}(x) V_{a}(0)= & (x \bar{x})^{a b} C_{+}^{\mathrm{L}}(a)\left[V_{a-b}\right]_{\mathrm{ee}} \\
& +(x \bar{x})^{1+b^{2}} \tilde{C}_{0}^{\mathrm{L}}(a)\left[V_{a}\right]_{\mathrm{oo}}+(x \bar{x})^{1-b a+b^{2}} C_{-}^{\mathrm{L}}(a)\left[V_{a+b}\right]_{\mathrm{ee}} \\
\Lambda_{1,3}(x) V_{a}(0)= & (x \bar{x})^{a b+1 / 2} C_{+}^{\mathrm{L}}(a)\left[V_{a-b}\right]_{\mathrm{oo}} \\
& +(x \bar{x})^{b^{2}} \tilde{C}_{0}^{\mathrm{L}}(a)\left[V_{a}\right]_{\mathrm{ee}}+(x \bar{x})^{1-b a+b^{2}} C_{-}^{\mathrm{L}}(a)\left[V_{a+b}\right]_{\mathrm{oo}}
\end{aligned}
$$

where $C_{-}(a), \tilde{C}_{0}(a)$, and $C_{+}(a)$ are "special" structure constants 4

$$
\begin{aligned}
& C_{+}^{\mathrm{L}}(a)=1 \\
& \tilde{C}_{0}^{\mathrm{L}}(a)=\frac{2 \pi i \mu \gamma\left(a b-b^{2}\right)}{\gamma\left(-b^{2}\right) \gamma(a b)} \\
& C_{-}^{\mathrm{L}}(a)=\pi^{2} \mu^{2} b^{4} \gamma^{2}\left(\frac{1}{2}+\frac{b^{2}}{2}\right) \gamma\left(-\frac{1}{2}-\frac{b^{2}}{2}+a b\right) \gamma\left(\frac{1}{2}-\frac{b^{2}}{2}-a b\right) .
\end{aligned}
$$

The analogous operator products in the matter sector can be easily reconstructed by starting from (62), renormalizing the fields, and analytically continuing, as discussed in Sec. 3,

$$
\begin{aligned}
\Phi_{13}(x) \Phi_{a}(0)= & (x \bar{x})^{1-a b-b^{2}} C_{-}^{\mathrm{M}}(a)\left[\Phi_{a-b}\right]_{\mathrm{ee}} \\
& +(x \bar{x})^{1-b^{2}} \tilde{C}_{0}^{\mathrm{M}}(a)\left[\Phi_{a}\right]_{\mathrm{oo}}+(x \bar{x})^{a b} C_{+}^{\mathrm{M}}(a)\left[\Phi_{a+b}\right]_{\mathrm{ee}} \\
\Psi_{13}(x) \Phi_{a}(0)= & (x \bar{x})^{1-a b-b^{2}} C_{-}^{\mathrm{M}}(a)\left[\Phi_{a-b}\right]_{\mathrm{oo}} \\
& +(x \bar{x})^{-b^{2}} \tilde{C}_{0}^{\mathrm{M}}(a)\left[\Phi_{a}\right]_{\mathrm{ee}}+(x \bar{x})^{a b} C_{+}^{\mathrm{M}}(a)\left[\Phi_{a+b}\right]_{\mathrm{oo}}
\end{aligned}
$$

and

$$
\begin{aligned}
& C_{-}^{\mathrm{M}}(a)=\left(\frac{\gamma\left(1 / 2+b^{2} / 2\right) \gamma\left(a b-1 / 2+b^{2} / 2\right)}{\gamma\left(-1 / 2+3 b^{2} / 2\right) \gamma\left(a b+1 / 2-b^{2} / 2\right)}\right)^{1 / 2}, \\
& \tilde{C}_{0}^{\mathrm{M}}(a)=i b^{-2} \gamma\left(\frac{b Q}{2}\right)\left(\frac{\gamma\left(1-b^{2}\right) \gamma\left(b^{2} / 2-1 / 2\right)}{\gamma\left(b^{2}-1\right) \gamma\left(3 b^{2} / 2-1 / 2\right)}\right)^{1 / 2} \frac{\gamma\left(a b+b^{2}\right)}{\gamma(a b)}, \\
& C_{+}^{\mathrm{M}}(a)=\left(\frac{\gamma\left(1 / 2+b^{2} / 2\right) \gamma\left(a b-1 / 2+3 b^{2} / 2\right)}{\gamma\left(-1 / 2+3 b^{2} / 2\right) \gamma\left(a b+1 / 2+b^{2} / 2\right)}\right)^{1 / 2} .
\end{aligned}
$$

\footnotetext{
${ }^{3}$ In the Liouville sector, our notation for the top component of the primary supermultiplet differs from the notation in $[10,11]$.

${ }^{4}$ Again, here we use the notation for the Liouville special structure constants differently (which seems more natural) than in $[10,11]$.
} 
It is straightforward to verify that we are left with

$$
O_{13}(x) W_{a}(0)=A_{0,-2}^{(1,3)} W_{a-b}(0)+A_{0,0}^{(1,3)} W_{a}(0)+A_{0,2}^{(1,3)} W_{a+b}(0)
$$

in the operator product (see Appendix A). The cancellation of the "unphysical" terms in the operator product can be verified by explicitly calculating at least at the primary field level. The coefficients can be written in the special factored form

$$
\begin{aligned}
& A_{0,-2}^{(1,3)}=\left(1-2 a b+b^{2}\right)^{2} C_{-}^{\mathrm{M}}(a-b) C_{+}^{\mathrm{L}}(a)=X \frac{N(a)}{N(a-b)}, \\
& A_{0,0}^{(1,3)}=\tilde{C}_{0}^{\mathrm{M}}(a-b) \tilde{C}_{0}^{\mathrm{L}}(a)=X \frac{N(a)}{N(a)}, \\
& A_{0,2}^{(1,3)}=\left(1-2 a b+b^{2}\right)^{2} C_{+}^{\mathrm{M}}(a-b) C_{-}^{\mathrm{L}}(a)=X \frac{N(a)}{N(a+b)},
\end{aligned}
$$

where

$$
X=4 b^{2}\left[\pi \mu \gamma\left(\frac{1}{2}+\frac{b^{2}}{2}\right)\right]\left[\frac{\gamma\left(1 / 2+b^{2} / 2\right)}{\gamma\left(3 b^{2} / 2-1 / 2\right)}\right]^{1 / 2}
$$

and

$$
N(a)=\left[\pi \mu \gamma\left(\frac{1}{2}+\frac{b^{2}}{2}\right)\right]^{-a / b}\left[\gamma\left(a b-\frac{b^{2}}{2}+\frac{1}{2}\right) \gamma\left(\frac{a}{b}-\frac{b^{-2}}{2}+\frac{1}{2}\right)\right]^{1 / 2} .
$$

A similar calculation can be performed for the operator product $O_{m, n} \tilde{W}$. It turns out that the result can be generalized for an arbitrary pair $(m, n)$ as

$$
A_{r, s}^{(m, n)}=\tilde{A}_{r, s}^{(m, n)}=K B_{m, n} N\left(a_{m,-n}\right) \frac{N(a)}{N\left(a+\lambda_{r, s}\right)},
$$

and the coefficient $K$ is universal, i.e., is independent of $(m, n)$,

$$
K=\frac{1}{2 b}\left[\frac{\gamma\left(1 / 2+b^{2} / 2\right)}{\gamma\left(3 / 2-b^{-2} / 2\right)}\right]^{1 / 2} .
$$

Expression (58) reduces to

$$
\begin{aligned}
& O_{m, n} \frac{W(a)}{N(a)}=K B_{m, n} N\left(a_{m,-n}\right) \sum_{\{r, s\} \in(m, n)} \frac{W\left(a+\lambda_{r, s}\right)}{N\left(a+\lambda_{r, s}\right)}, \\
& O_{m, n} \frac{\tilde{W}(a)}{N(a)}=K B_{m, n} N\left(a_{m,-n}\right) \sum_{\{r, s\} \in(m, n)} \frac{\tilde{W}\left(a+\lambda_{r, s}\right)}{N\left(a+\lambda_{r, s}\right)} .
\end{aligned}
$$




\section{Correlation numbers}

The requirement that the ghost current be conserved leads to the general form of the $n$-point correlation numbers on the sphere for the three basic types of observables introduced in the previous section:

$$
\left\langle\left\langle a_{1} a_{2} \cdots a_{n}\right\rangle\right\rangle_{\mathrm{SLG}}=\left\langle W_{a_{1}}\left(z_{1}\right) W_{a_{2}}\left(z_{2}\right) \tilde{W}_{a_{3}}\left(z_{3}\right) \prod_{i=4}^{n} \int \bar{G}_{-1 / 2} G_{-1 / 2} U_{a_{i}}\left(z_{i}\right) d^{2} z_{i}\right\rangle .
$$

The simplest case is the three-point correlation number, where there is no integral over the moduli space and the result is factored into a product of the matter, Liouville, and ghost three-point functions. Using (47) and (49), we can derive

$$
\begin{aligned}
\left\langle\left\langle a_{1} a_{2} a_{3}\right\rangle\right\rangle_{\mathrm{SLG}}= & {\left[\left\langle V_{a_{1}} V_{a_{2}} V_{a_{3}}\right\rangle\left\langle\Phi_{a_{1}-b} \Phi_{a_{2}-b} \Psi_{a_{3}-b}\right\rangle\right.} \\
& \left.+\left\langle V_{a_{1}} V_{a_{2}} \Lambda_{a_{3}}\right\rangle\left\langle\Phi_{a_{1}-b} \Phi_{a_{2}-b} \Phi_{a_{3}-b}\right\rangle\right]\left\langle c_{1} c_{2} c_{3}\right\rangle\left\langle\delta\left(\gamma_{1}\right) \delta\left(\gamma_{2}\right)\right\rangle \\
= & C^{\mathrm{M}}\left(a_{1}-b, a_{2}-b, a_{3}-b\right) \tilde{C}^{\mathrm{L}}\left(a_{1}, a_{2}, a_{3}\right) \\
& +\tilde{C}^{\mathrm{M}}\left(a_{1}-b, a_{2}-b, a_{3}-b\right) C^{\mathrm{L}}\left(a_{1}, a_{2}, a_{3}\right)
\end{aligned}
$$

or explicitly (see Appendix E)

$$
\left\langle\left\langle a_{1} a_{2} a_{3}\right\rangle\right\rangle_{\mathrm{SLG}}=\Omega \prod_{i=1}^{3} N\left(a_{i}\right)
$$

where

$$
\Omega=i\left[\pi \mu \gamma\left(\frac{1}{2}+\frac{b^{2}}{2}\right)\right]^{Q / b}\left[\frac{\gamma\left(b^{2} / 2+1 / 2\right) \gamma\left(b^{-2} / 2-1 / 2\right)}{b^{2}}\right]^{1 / 2}
$$

and the normalization factor $N(a)$ is defined in (69).

The partition sum and the two-point numbers can be obtained simply from the expression for the three-point function. The next step is to obtain the four-point numbers. The expression is more complicated: it involves integration over moduli. The naive and rather numerical way to calculate it is based on the conformal block decomposition of the fourpoint correlation functions in both the Liouville and the matter sectors (see, [10,27, 28]). But this approach is not available at the moment (we intend to investigate it later), and this straightforward computation is moreover unable to provide the exact results. Here, we use another approach to evaluate the four-point integral. Relation (57) allows reducing the moduli integral in expression (73) for the correlation numbers to the boundary integrals if one of the parameters is degenerate, $a_{i}=a_{m,-n}$. In particular, the four-point correlation number becomes

$$
\left\langle\left\langle a_{m,-n} a_{1} a_{2} a_{3}\right\rangle\right\rangle_{\mathrm{SLG}}=B_{m, n}^{-1} \int_{\partial \Gamma} \partial\left\langle O_{m, n}^{\prime}(x) W_{a_{1}}\left(x_{1}\right) W_{a_{2}}\left(x_{2}\right) \tilde{W}_{a_{3}}\left(x_{3}\right)\right\rangle \frac{d x}{2 i},
$$

where the boundary consists of three circles $\partial \Gamma=\sum_{i=1}^{3} \partial \Gamma_{i}$ around the points $x_{1}, x_{2}$, and $x_{3}$ (integrated clockwise) and a large circle $\partial \Gamma_{\infty}$ near infinity (integrated counterclockwise), 
which arises because the operator $O_{m, n}^{\prime}$ is not exactly a scalar. Taking the distributive character of the action of the BRST charge into account, we can shift it after the insertion of the other physical fields, which means that $Q$-exact terms in (57) do not contribute to (77). To evaluate the boundary terms, we must better understand the short-range behavior of the operator products $O_{m, n}^{\prime}(x) W(0)$ and $O_{m, n}^{\prime}(x) \tilde{W}(0)$. The next section is devoted to this subject.

\section{Boundary terms}

The boundary integrals in (77) are controlled by the operator products of the logarithmic fields $O_{m, n}^{\prime}$ with the basic physical states $W\left(a_{i}\right)$ and $\tilde{W}\left(a_{i}\right)$. The derivation of these OPEs on the basis of the corresponding ground ring operator products is almost the same as in the bosonic case $[29,30]$. The only differences are the different definition of the logarithmic field $V^{\prime}(a)$ and the different relation between the conformal dimension and the parameter $b$. A closer consideration shows that these two differences compensate each other. Hence, the necessary logarithmic contributions to the OPE are

$$
\begin{aligned}
& O_{m, n}^{\prime} \frac{W(a)}{N(a)}=\log (x \bar{x}) K B_{m, n} N\left(a_{m,-n}\right) \sum_{\{r, s\} \in(m, n)} q_{r, s}^{(m, n)}(a) \frac{W\left(a+\lambda_{r, s}\right)}{N\left(a+\lambda_{r, s}\right)}, \\
& O_{m, n}^{\prime} \frac{\tilde{W}(a)}{N(a)}=\log (x \bar{x}) K B_{m, n} N\left(a_{m,-n}\right) \sum_{\{r, s\} \in(m, n)} q_{r, s}^{(m, n)}(a) \frac{\tilde{W}\left(a+\lambda_{r, s}\right)}{N\left(a+\lambda_{r, s}\right)},
\end{aligned}
$$

where

$$
q_{r, s}^{(m, n)}(a)=\left|a-\lambda_{r, s}-Q / 2\right|_{\operatorname{Re}}-\lambda_{m, n}
$$

and

$$
|x|_{\operatorname{Re}}=\left\{\begin{aligned}
x & \text { if } \operatorname{Re} x>0 \\
-x & \text { if } \operatorname{Re} x<0
\end{aligned}\right.
$$

The contribution at infinity arises as follows. The logarithmic field is not a scalar; under conformal coordinate transformations $x \rightarrow y$, it acquires an inhomogeneous part

$$
O_{m, n}^{\prime}(y)=O_{m, n}^{\prime}(x)-2 \Delta_{m, n}^{\prime} O_{m, n}(x) \log \left|y_{x}\right|,
$$

where

$$
\Delta_{m, n}^{\prime}=\left.\frac{d}{d a} \Delta_{a}^{(\mathrm{L})}\right|_{a=a_{m, n}}=\lambda_{m, n}
$$

Transformation (81) leads to the behavior of the correlation function with $O_{m, n}^{\prime}(x)$ as $x \rightarrow \infty$

$$
\left\langle O_{m, n}^{\prime}(x) W_{a_{1}}\left(x_{1}\right) W_{a_{2}}\left(x_{2}\right) \tilde{W}_{a_{3}}\left(x_{3}\right)\right\rangle \sim-2 \Delta_{m, n}^{\prime} \log (x \bar{x})\left\langle O_{m, n} W_{a_{1}} W_{a_{2}} \tilde{W}_{a_{3}}\right\rangle .
$$

Therefore, the contribution of the boundary term $\partial \Gamma_{\infty}$ is evaluated as

$$
\frac{1}{2 i} \int_{\partial \Gamma_{\infty}} \partial\left\langle O_{m, n}^{\prime}(x) W_{a_{1}}\left(x_{1}\right) W_{a_{2}}\left(x_{2}\right) \tilde{W}_{a_{3}}\left(x_{3}\right)\right\rangle d x=-2 \pi \lambda_{m, n}\left\langle O_{m, n} W_{a_{1}} W_{a_{2}} \tilde{W}_{a_{3}}\right\rangle .
$$




\section{Four-point correlation number}

Summing boundary contributions (78) and curvature term (84), we find the expression for the four-point correlation number

$$
\left\langle\left\langle a_{m,-n} a_{1} a_{2} a_{3}\right\rangle\right\rangle_{\mathrm{SLG}}=\pi K N\left(a_{m,-n}\right)\left\{\sum_{i=1}^{3} \sum_{r, s \in(m, n)} q_{r, s}^{(m, n)}\left(a_{i}\right)+2 m n \lambda_{m, n}\right\}\left\langle\left\langle a_{1} a_{2} a_{3}\right\rangle\right\rangle
$$

where the fusion set $(m, n)=\{1-m: 2: m-1,1-n: 2: n-1\}$ and the second term is just the result of the curvature contribution when we move $O_{m, n}^{\prime}$ close to one of the other fields. The normalization factor $N\left(a_{m,-n}\right)$ is defined by (69), and the three-point correlation number $\left\langle\left\langle a_{1} a_{2} a_{3}\right\rangle\right\rangle$ is given by $(75)$. It seems tempting to simplify these relations by introducing the renormalized fields $\mathcal{W}_{a}, \tilde{\mathcal{W}}_{a}$, and $\mathcal{U}_{a}$ as

$$
\begin{aligned}
& \mathcal{W}_{a}=\frac{W_{a}}{N(a)}, \\
& \tilde{\mathcal{W}}_{a}=\frac{\tilde{W}_{a}}{N(a)}, \\
& \mathcal{U}_{a}=\frac{U_{a}}{N(a)} .
\end{aligned}
$$

Expression (85) reduces to

$$
\begin{array}{r}
\frac{1}{\Omega} \int\left\langle\bar{G}_{-1 / 2} G_{-1 / 2} \mathcal{U}_{m,-n}(x) \mathcal{W}_{a_{1}}\left(x_{1}\right) \mathcal{W}_{a_{2}}\left(x_{2}\right) \tilde{\mathcal{W}}_{a_{3}}\left(x_{3}\right)\right\rangle d^{2} x \\
=\pi K\left\{\sum_{i=1}^{3} \sum_{r, s \in(m, n)} q_{r, s}^{(m, n)}\left(a_{i}\right)+2 m n \lambda_{m, n}\right\}
\end{array}
$$

where $\Omega$ is defined in (76). The formulas (85) and (87) for the four-point correlation numbers in the minimal super Liouville gravity, together with the explicit expression for the structure constants (75), are the main results of the presented study.

Acknowledgments Authors are grateful to M. Bershtein, D. Friedan, A. Litvinov, A. Losev and D. Polyakov for the useful discussions. A. B. was supported by the RAS program "Elementary Particles and Fundamental Nuclear Physics" and the Russian Foundation for Basic Research (Grant No. 07-02-00799) and also by Grant No. SS-3472.2008.2. V. B. was supported by the Russian Foundation for Basic Research (Grant No. 08-01-00720). V. B. sincerely thanks A. Neveu and other members of LPTA, Montpellier University II, for their hospitality and the interest in this work.

Appendix A. Ghost number balance on the sphere.

Conservation of the fermionic ghost current provides the relation

$$
\oint_{C} \frac{d u}{2 \pi i}\left\langle J^{b c}(u) X\left(z_{1}, \ldots, z_{n}\right)\right\rangle=\left(N_{c}-N_{b}\right)\left\langle X\left(z_{1}, \ldots, z_{n}\right)\right\rangle,
$$


where $X\left(z_{1}, \ldots, z_{n}\right)$ denotes a set of the physical fields, the contour $C$ is any contour encircling all insertions of the fields, and $N_{c}$ and $N_{b}$ are the total ghost numbers related to the composite operator $X$. On the other hand, we can deform the contour, moving it to infinity. For this, we need to know the transformation law for $J^{b c}$. First, we define the infinitesimal version. Based on the canonical operator products

$$
\begin{aligned}
& T(u) b(z)=\frac{2}{(u-z)^{2}} b(z)+\frac{1}{u-z} b^{\prime}(z), \\
& T(u) c(z)=\frac{-1}{(u-z)^{2}} c(z)+\frac{1}{u-z} c^{\prime}(z),
\end{aligned}
$$

correspond to the following ghost part of the stress-energy tensor

$$
T^{b c}(u)=: c(u) b(u):+2: \partial c(u) b(u):
$$

which defines (see (24)) the singular part of the operator product

$$
T^{b c}(u) J^{b c}(z)=-\frac{3}{(u-z)^{3}}+\frac{J^{b c}(z)}{(u-z)^{2}}+\frac{\partial J^{b c}(z)}{(u-z)} .
$$

This, together with the definition

$$
\delta_{\epsilon}=\oint \epsilon(u) T(u) \frac{d u}{2 \pi i}
$$

leads to

$$
\delta_{\epsilon} J^{b c}(z)=-\frac{3}{2} \epsilon^{\prime \prime}(z)+\epsilon^{\prime}(z) J^{b c}(z)+\epsilon \frac{\partial J^{b c}(z)}{\partial z} .
$$

This infinitesimal form allows reconstructing the finite version of the transformation

$$
J^{b c}(z) \rightarrow \tilde{J}^{b c}(z)=\frac{d w}{d z} J^{b c}(w(z))-\frac{3}{2} \frac{w^{\prime \prime}}{w^{\prime}} \quad \text { as } z \rightarrow w(z) .
$$

Also taking the transformation properties of the physical fields into account, we now make the inversion for the correlation function in (88),

$$
\begin{aligned}
\oint_{C} \frac{d u}{2 \pi i}\left\langle J^{b c}(u) X\right\rangle & =\oint_{C_{\infty}} \frac{d u}{2 \pi i}\left[-\frac{1}{u^{2}}\left\langle J^{b c}(1 / u) X\left(1 / z_{1}, \ldots, 1 / z_{n}\right)\right\rangle+\frac{3}{u}\left\langle X\left(1 / z_{1}, \ldots, 1 / z_{n}\right)\right\rangle\right] \\
& =3\left\langle X\left(1 / z_{1}, \ldots, 1 / z_{n}\right)\right\rangle
\end{aligned}
$$

where the first term vanishes because $1 / u=0$ is the regular point of the correlation function. Again performing the inversion for the correlator $\left\langle X\left(1 / z_{1}, \ldots, 1 / z_{n}\right)\right\rangle$, we obtain

$$
\left(N_{c}-N_{b}-3\right)\left\langle X\left(z_{1}, \ldots, z_{n}\right)\right\rangle=0,
$$

which means that either $N_{c}-N_{b}=3$ or the corresponding correlator is equal to zero. 
The consideration for the bosonic ghost current $J^{\beta \gamma}(z)$ literally follows that for the fermionic ghost current. We consider the integral

$$
\begin{aligned}
& \oint_{C} \frac{d u}{2 \pi i}\left\langle J^{\beta \gamma}(u) X\left(z_{1}, \ldots, z_{n}\right)\right\rangle=\left(-N_{\delta(\gamma)}+N_{\gamma}+N_{\delta(\beta)}-N_{\beta}\right)\left\langle X\left(z_{1}, \ldots, z_{n}\right)\right\rangle, \\
& T^{\beta \gamma}(u)=-\frac{1}{2}: \beta^{\prime}(u) \gamma(u):-\frac{3}{2}: \gamma^{\prime}(u) \beta(u):
\end{aligned}
$$

and

$$
T^{\beta \gamma}(u) J^{\beta \gamma}(z)=\frac{2}{(u-z)^{3}}+\frac{J^{\beta \gamma}(z)}{(u-z)^{2}}+\frac{\partial J^{\beta \gamma}(z)}{(u-z)} .
$$

Hence, the infinitesimal form of the transformation is

$$
\delta_{\epsilon} J^{\beta \gamma}(z)=\epsilon^{\prime \prime}(z)+\epsilon^{\prime}(z) J^{\beta \gamma}(z)+\epsilon \frac{\partial J^{\beta \gamma}(z)}{\partial z}
$$

and the finite version is

$$
J^{\beta \gamma}(z) \rightarrow \tilde{J}^{\beta \gamma}(z)=\frac{d w}{d z} J^{\beta \gamma}(w(z))+\frac{w^{\prime \prime}}{w^{\prime}} \quad \text { as } z \rightarrow w(z) .
$$

The only difference comes from the different coefficient in the transformation law. We conclude that

$$
\left(-N_{\delta(\gamma)}+N_{\gamma}+N_{\delta(\beta)}-N_{\beta}+2\right)\left\langle X\left(z_{1}, \ldots, z_{n}\right)\right\rangle=0
$$

\section{Appendix B. BRST properties of the field $\tilde{W}_{a}$.}

Here, we prove that $\tilde{W}_{a}$ is closed. We must verify that

$$
Q \tilde{W}_{a}=Q\left(G_{-1 / 2}^{\mathrm{M}+\mathrm{L}}+\frac{1}{2} G_{-1 / 2}^{g}\right)\left|\Omega_{a}\right\rangle_{0}=Q\left(G_{-1 / 2}^{\mathrm{M}+\mathrm{L}}-b_{-1} \gamma_{1 / 2}\right)\left|\Omega_{a}\right\rangle_{0}=0
$$

where the second equality follows from the mode expansion of $G_{1 / 2}^{g}$. In accordance with definition (41), we split operator $Q$ into three parts $Q_{1}, Q_{2}$, and $Q_{3}$. Then

$$
\begin{aligned}
Q_{1} G_{-1 / 2}^{\mathrm{M}+\mathrm{L}}\left|\Omega_{a}\right\rangle_{0} & =\frac{1}{2} c_{0} G_{-1 / 2}\left|\Omega_{a}\right\rangle_{0}, \\
Q_{2} G_{-1 / 2}^{\mathrm{M}+\mathrm{L}}\left|\Omega_{a}\right\rangle_{0} & =\left(\gamma_{1 / 2} L_{-1}+\gamma_{-1 / 2}+\gamma_{1 / 2}^{2} G_{-1 / 2} b_{-1}-\frac{1}{4} c_{0} G_{-1 / 2}\right)\left|\Omega_{a}\right\rangle_{0}, \\
Q_{3} G_{-1 / 2}^{\mathrm{M}+\mathrm{L}}\left|\Omega_{a}\right\rangle_{0} & =-\frac{1}{4} c_{0} G_{-1 / 2}\left|\Omega_{a}\right\rangle_{0} .
\end{aligned}
$$

The sum of these contributions is

$$
Q G_{-1 / 2}^{\mathrm{M}+\mathrm{L}}\left|\Omega_{a}\right\rangle_{0}=\left(\gamma_{1 / 2} L_{-1}+\gamma_{-1 / 2}+\gamma_{1 / 2}^{2} G_{-1 / 2} b_{-1}\right)\left|\Omega_{a}\right\rangle_{0}
$$


Similarly, the action of the operator $Q$ on the second term in $\tilde{W}_{a}$ is

$$
\begin{aligned}
Q_{1} b_{-1} \gamma_{1 / 2}\left|\Omega_{a}\right\rangle_{0} & =\left(\gamma_{1 / 2} L_{-1}+\frac{1}{2} \gamma_{-1 / 2}+\frac{3}{4} c_{0} b_{-1} \gamma_{1 / 2}\right)\left|\Omega_{a}\right\rangle_{0} \\
Q_{2} b_{-1} \gamma_{1 / 2}\left|\Omega_{a}\right\rangle_{0} & =\left(\gamma_{1 / 2}^{2} G_{-1 / 2} b_{-1}+\frac{1}{2} \gamma_{-1 / 2}-\frac{1}{2} c_{0} b_{-1} \gamma_{1 / 2}\right)\left|\Omega_{a}\right\rangle_{0} \\
Q_{3} b_{-1} \gamma_{1 / 2}\left|\Omega_{a}\right\rangle_{0} & =-\frac{1}{4} c_{0} b_{-1} \gamma_{1 / 2}\left|\Omega_{a}\right\rangle_{0}
\end{aligned}
$$

Again summing these contributions, we obtain

$$
Q b_{-1} \gamma_{1 / 2}\left|\Omega_{a}\right\rangle_{0}=\left(\gamma_{1 / 2} L_{-1}+\gamma_{-1 / 2}+\gamma_{1 / 2}^{2} G_{-1 / 2} b_{-1}\right)\left|\Omega_{a}\right\rangle_{0}
$$

This coincides with the the action of the BRST charge on the first term (105). We have thus verified that fields $\tilde{W}_{a}$ form the cohomology class.

\section{Appendix C. The basic relation}

We first consider the action of the holomorphic BRST charge on the discrete state $O_{m, n}$. We assume that we are dealing with the Verma module in the Liouville sector (i.e., the degenerate representation is not factored with respect to the singular vector submodule). From dimensional arguments and also taking the ghost charge of the operator $Q$ into account, we can conclude that the most general form of this action is

$$
Q O_{m, n}=\left(x G_{-1 / 2}^{\mathrm{M}}+y G_{-1 / 2}^{\mathrm{L}}+z G_{-1 / 2}^{\mathrm{g}}\right) \Phi_{m, n} D_{m, n}^{\mathrm{L}} V_{m, n} c .
$$

Indeed, decoupling the singular vector then provides that $O_{m, n}$ is BRST exact. The explicit calculation gives $x=y=1$ and $z=-1 / 2$. Combining with the action of the antiholomorhic $\bar{Q}$, we obtain basic relation (53). We now consider the "quasi"-discrete state

$$
O_{a}=\bar{H}_{m, n} H_{m, n} \Phi_{m, n} V_{a}
$$

for which the parameter $a$ is in the vicinity of the degenerate value or, more precisely, the difference $\epsilon=a-a_{m, n}$ is small. It is obvious from the analyticity that

$$
\bar{Q} Q O_{a}=\left[\bar{J}_{m, n}+\epsilon \bar{K}_{m, n}\right]\left[J_{m, n}+\epsilon K_{m, n}\right] \Phi_{m, n} V_{a}
$$

where $J_{m, n}$ is defined in (52) and $K_{m, n}$ is an operator built from the super Virasoro generators of all three sectors. Differentiating (110) with respect to the parameter $a$ gives

$$
\begin{aligned}
\bar{Q} Q O_{a}^{\prime} & =\bar{J}_{m, n} J_{m, n} \Phi_{m, n} V_{m, n}^{\prime} \\
& =\left(\bar{G}_{-1 / 2}^{\mathrm{M}+\mathrm{L}}-\frac{1}{2} \bar{G}_{-1 / 2}^{\mathrm{g}}\right)\left(G_{-1 / 2}^{\mathrm{M}+\mathrm{L}}-\frac{1}{2} G_{-1 / 2}^{\mathrm{g}}\right) \Phi_{m, n} \bar{D}_{m, n}^{\mathrm{L}} D_{m, n}^{\mathrm{L}} V_{m, n}^{\prime} \bar{c} c
\end{aligned}
$$

because the term

$$
\left(\bar{K}_{m, n} J_{m, n}+K_{m, n} \bar{J}_{m, n}\right) \Phi_{m, n} V_{m, n}
$$


vanishes as a result of the action of the operators $D_{m, n}^{L}$ and $\bar{D}_{m, n}^{L}$ inside $J_{m, n}$ and $\bar{J}_{m, n}$ on $V_{m, n}$. Relation (111) combined with higher equations of motion (14) results in (54).

Appendix D. OPE $O_{1,3} W_{a}$.

We separately calculate the contribution of each term in (60) to the operator product $O_{1,3}(x) W_{a}(0)$. The first term gives (we write only the holomorphic part explicitly)

$$
\begin{aligned}
\Phi_{13}^{\prime}( & x) V_{13}(x) \Phi_{a-b}(0) V_{a}(0) c(0) \delta(\gamma(0)) \\
= & \left(x^{1-a b} C_{+}^{\mathrm{M}}(a-b) \Phi_{a-2 b}(0)+x^{1-b^{2}} \tilde{C}_{0}^{\mathrm{M}}(a-b) \Psi_{a-b}(0)+x^{a b-b^{2}} C_{-}^{\mathrm{M}}(a-b) \Phi_{a}(0)\right)^{\prime} \\
& \times\left(x^{a b} C_{-}^{\mathrm{L}}(a) V_{a-b}(0)+x^{1+b^{2}} \tilde{C}_{0}^{\mathrm{L}}(a) \Lambda_{a-b}(0)+x^{1-a b+b^{2}} C_{+}^{\mathrm{L}}(a) V_{a+b}(0)\right) C(0) \delta(\gamma(0)) \\
= & (1-a b) C_{+}^{(M)}(a-b) C_{-}(a) W_{a-b}(0)+\left(a b-b^{2}\right) C_{-}^{(M)}(a-b) C_{+}(a) W_{a+b}(0) .
\end{aligned}
$$

The contribution of the second term in (60) is

$$
\begin{aligned}
& \Phi_{13}(x) V_{13}^{\prime}(x) \Phi_{a-b}(0) V_{a}(0) C(0) \delta(\gamma(0)) \\
& \quad=a b C_{+}^{\mathrm{M}}(a-b) C_{-}^{\mathrm{L}}(a) W_{a-b}(0)+\left(1-a b+b^{2}\right) C_{-}^{\mathrm{M}}(a-b) C_{+}^{\mathrm{L}}(a) W_{a+b}(0) .
\end{aligned}
$$

The third term contributes

$$
\Psi_{13}(x) \Lambda_{13}(x) \Phi_{a-b}(0) V_{a}(0) c(0) \delta(\gamma(0))=\tilde{C}_{0}^{\mathrm{M}}(a-b) \tilde{C}_{0}^{\mathrm{L}}(a) W_{a}(0)
$$

Using the basic operator product in the ghost sector, we obtain

$$
\beta(x) \delta(\gamma(0))=\frac{\delta^{\prime}(\gamma(0))}{x}
$$

and

$$
\gamma(x) \delta^{\prime}(\gamma(0)) \sim \gamma(0) \delta^{\prime}(\gamma(0)) \sim-\delta(\gamma(0)) .
$$

Hence, the fourth term contributes

$$
\begin{aligned}
& \Phi_{13}(x) \Phi_{a-b}(0) V_{13}(x) V_{a}(0) \beta(x) \gamma(x) c(0) \delta(\gamma(0))= \\
& \quad-C_{+}^{\mathrm{M}}(a-b) C_{-}^{\mathrm{L}}(a) W_{a-b}(0)-C_{-}^{\mathrm{M}}(a-b) C_{+}^{\mathrm{L}}(a) W_{a+b}(0) .
\end{aligned}
$$

The last term contributes

$$
\begin{aligned}
& \Phi_{13}(x) \Phi_{a-b}(0) V_{13}(x) V_{a}(0) b(x) c(x) c(0) \delta(\gamma(0))= \\
& \quad C_{+}^{\mathrm{M}}(a-b) C_{-}^{\mathrm{L}}(a) W_{a-b}(0)+C_{-}^{\mathrm{M}}(a-b) C_{+}^{\mathrm{L}}(a) W_{a+b}(0) .
\end{aligned}
$$

Combining all together and also taking the antiholomorphic part into account, we obtain (66).

\section{Appendix E. Three-point correlation number in SLG}

Here, we explicitly derive three-point number (74),

$$
\left\langle\left\langle a_{1} a_{2} a_{3}\right\rangle\right\rangle_{\mathrm{SLG}}=C_{\mathrm{I}}^{\mathrm{SLG}}\left(a_{1}, a_{2}, a_{3}\right)+C_{\mathrm{II}}^{\mathrm{SLG}}\left(a_{1}, a_{2}, a_{3}\right),
$$


where

$$
\begin{aligned}
& C_{\mathrm{I}}^{\mathrm{SLG}}\left(a_{1}, a_{2}, a_{3}\right)=C^{\mathrm{L}}\left(a_{1}, a_{2}, a_{3}\right) \tilde{C}^{\mathrm{M}}\left(a_{1}-b, a_{2}-b, a_{3}-b\right), \\
& C_{\mathrm{II}}^{\mathrm{SLG}}\left(a_{1}, a_{2}, a_{3}\right)=\tilde{C}^{\mathrm{L}}\left(a_{1}, a_{2}, a_{3}\right) C^{\mathrm{M}}\left(a_{1}-b, a_{2}-b, a_{3}-b\right) .
\end{aligned}
$$

Instead of treating the two sectors separately, we find it instructive to solve the shift relations for the SLG structure constants. Below, we show that

$$
\begin{aligned}
\frac{C_{\mathrm{I}, \mathrm{II}}^{\mathrm{SLG}}\left(a_{1}+b, a_{2}, a_{3}\right)}{C_{\mathrm{I}, \mathrm{II}}^{\mathrm{SLG}}\left(a_{1}-b, a_{2}, a_{3}\right)}= & {\left[\pi \mu b^{2} \gamma\left(1 / 2+b^{2} / 2\right)\right]^{-2}\left(1 / 2-b a+b^{2} / 2\right)\left(1 / 2-b a-b^{2} / 2\right) } \\
& \times\left[\frac{\gamma\left(a b+b^{2} / 2+1 / 2\right)}{\gamma\left(a b-3 b^{2} / 2+1 / 2\right)}\right]^{1 / 2} \cdot
\end{aligned}
$$

These shift relations give

$$
\left\langle\left\langle a_{1} a_{2} a_{3}\right\rangle\right\rangle_{\mathrm{SLG}}=\mathrm{const} \prod_{i=1}^{3} N\left(a_{i}\right),
$$

where $N(a)$ is defined in Eq. (69). To define the constant, we take $a_{i}=b$. The corresponding structure constants in the matter sector are $C^{\mathrm{M}}(0,0,0)=1$ and $\tilde{C}^{\mathrm{M}}(0,0,0)=0$. Also having in mind that $C^{\mathrm{L}}(b, b, b)$ can be defined from (9) and is expressed just in terms of the gamma functions, we conclude that const $=\Omega(b)$ (see Eq. (76)). The shift relations for SLG structure constants (122) are combined from the corresponding shift relations in the Liouville and the matter sectors,

$$
\frac{C_{\mathrm{I}}^{\mathrm{SLG}}\left(a_{1}+b, a_{2}, a_{3}\right)}{C_{\mathrm{I}}^{\mathrm{SLG}}\left(a_{1}-b, a_{2}, a_{3}\right)}=\frac{C^{\mathrm{L}}\left(a_{1}+b, a_{2}, a_{3}\right)}{C^{\mathrm{L}}\left(a_{1}-b, a_{2}, a_{3}\right)} \frac{\tilde{C}^{\mathrm{M}}\left(a_{1}, a_{2}-b, a_{3}-b\right)}{\tilde{C}^{\mathrm{M}}\left(a_{1}-2 b, a_{2}-b, a_{3}-b\right)},
$$

which follows from the bootstrap relations and the monodromy properties of the differential equation corresponding to the decoupling of the singular vector. For the sake of completeness, we recapitulate the two main formulas concerning the Liouville sector in $[10,11]$. The first is

$$
\begin{aligned}
\frac{C_{-}^{\mathrm{L}}\left(a_{1}\right) C_{a_{1}+b, a_{2}, a_{3}}^{\mathrm{L}}}{C_{+}^{\mathrm{L}}\left(a_{1}\right) C_{a_{1}-b, a_{2}, a_{3}}^{\mathrm{L}}}= & -\frac{\gamma\left(b a_{1}\right) \gamma\left(b a_{1}-b^{2}\right) \gamma^{2}\left(1 / 2-b^{2} / 2+b a_{1}\right)}{\left(1 / 2-b a_{1}+b^{2} / 2\right)^{2}} \\
& \times \frac{\gamma\left(1 / 2+b a_{2+3-1} / 2\right) \gamma\left(b a_{2+3-1} / 2-b^{2} / 2\right)}{\gamma\left(1 / 2+b a_{1+3-2} / 2\right) \gamma\left(b a_{1+3-2} / 2-b^{2} / 2\right)} \\
& \times \frac{\gamma\left(3 / 2-b a_{1+2+3} / 2+b^{2}\right) \gamma\left(1-b a_{1+2+3} / 2+b^{2} / 2\right)}{\gamma\left(1 / 2+b a_{1+2-3} / 2\right) \gamma\left(b a_{1+2-3} / 2-b^{2} / 2\right)},
\end{aligned}
$$


and the second is

$$
\begin{aligned}
\frac{C_{-}^{\mathrm{L}}\left(a_{1}\right) \tilde{C}^{\mathrm{L}}\left(a_{1}+b, a_{2}, a_{3}\right)}{C_{+}^{\mathrm{L}}\left(a_{1}\right) \tilde{C}^{\mathrm{L}}\left(a_{1}-b, a_{2}, a_{3}\right)}= & \frac{\gamma\left(b a_{1}\right) \gamma\left(b a_{1}-b^{2}\right)}{\gamma\left(b a_{1+2-3} / 2\right) \gamma\left(b a_{1-2+3} / 2\right)} \\
& \times \frac{\gamma\left(a_{1} b-b^{2} / 2+1 / 2\right) \gamma\left(a_{1} b-b^{2} / 2-1 / 2\right)}{\gamma\left(b a_{1+2+3} / 2-b^{2}\right) \gamma\left(\left(1+b a_{1-2+3}-b^{2}\right) / 2\right)} \\
& \times \frac{\gamma\left(b a_{-1+2+3} / 2\right) \gamma\left(\left(1+b a_{-1+2+3}-b^{2}\right) / 2\right)}{\gamma\left(\left(-1+b a_{1+2+3}-b^{2}\right) / 2\right) \gamma\left(\left(1+b a_{1+2-3}-b^{2}\right) / 2\right)} \\
= & S\left(a_{1}, a_{2}, a_{3}\right) .
\end{aligned}
$$

Taking (63) into account, we derive

$$
\begin{aligned}
\frac{C^{\mathrm{L}}\left(a_{1}+b, a_{2}, a_{3}\right)}{C^{\mathrm{L}}\left(a_{1}-b, a_{2}, a_{3}\right)}= & {\left[\pi \mu b^{2} \gamma\left(\frac{Q b}{2}\right)\right]^{-2} \frac{\gamma\left(b a_{1}\right) \gamma\left(b a_{1}-b^{2}\right)}{\gamma\left(1 / 2+b a_{1+3-2} / 2\right)} } \\
& \times \frac{\gamma\left(a_{1} b+b^{2} / 2+1 / 2\right) \gamma\left(a_{1} b-b^{2} / 2+1 / 2\right)}{\gamma\left(b a_{1+3-2} / 2-b^{2} / 2\right)} \\
& \times \frac{\gamma\left(1-b a_{1+2+3} / 2+b^{2} / 2\right) \gamma\left(3 / 2-b a_{1+2+3} / 2+b^{2}\right)}{\gamma\left(1 / 2+b a_{1+2-3} / 2\right)} \\
& \times \frac{\gamma\left(1 / 2+b a_{2+3-1} / 2\right) \gamma\left(b a_{2+3-1} / 2-b^{2} / 2\right)}{\gamma\left(b a_{1+2-3} / 2-b^{2} / 2\right)}
\end{aligned}
$$

from (125). The shift relations for the structure constants in the matter sector can be derived in two steps starting from those in the Liouville sector. First, we change the normalization in accordance with the standard requirement $\left\langle\Phi_{a} \Phi_{a}\right\rangle=1$ in the matter sector. In this normalization, the special structure constants coincide with the three-point functions (to distinguish these structure constants from the original ones, we do not label them with the superscript L):

$$
\begin{aligned}
& C_{-}(a)=C(-b, a, a+b), \\
& C_{+}(a)=C(-b, a, a-b) .
\end{aligned}
$$

Setting $a_{2}=a_{1}$ and $a_{3}=-b$ in (125), we obtain

$$
\left[\frac{C_{-}\left(a_{1}\right)}{C_{+}\left(a_{1}\right)}\right]^{2}=\frac{\gamma\left(a_{1} b-b^{2} / 2+1 / 2\right) \gamma\left(-a_{1} b+3 b^{2} / 2+3 / 2\right)}{\gamma\left(-a_{1} b+b^{2} / 2+3 / 2\right) \gamma\left(a_{1} b+b^{2} / 2+1 / 2\right)}=M\left(a_{1}\right) .
$$

Combining this relation with (126), we derive

$$
\frac{\tilde{C}\left(a_{1}+b, a_{2}, a_{3}\right)}{\tilde{C}\left(a_{1}-b, a_{2}, a_{3}\right)}=S\left(a_{1}, a_{2}, a_{3}\right) M^{-1 / 2}\left(a_{1}\right) .
$$


The second step now is to replace $a_{i} \rightarrow i\left(a_{i}-b\right)$ and $b \rightarrow-i b$,

$$
\begin{aligned}
\frac{\tilde{C}^{\mathrm{M}}\left(a_{1}, a_{2}-b, a_{3}-b\right)}{\tilde{C}^{\mathrm{M}}\left(a_{1}-2 b, a_{2}-b, a_{3}-b\right)}= & \frac{M^{1 / 2}\left(i\left(a_{1}-b\right) ;-i b\right)}{S\left(i\left(a_{1}-b\right), i\left(a_{2}-b\right), i\left(a_{3}-b\right) ;-i b\right)} \\
= & \frac{\gamma\left(a_{1} b-b^{2} / 2+1 / 2\right) \gamma\left(-a_{1} b-b^{2} / 2+3 / 2\right)}{\gamma\left(-a_{1} b+b^{2} / 2+3 / 2\right) \gamma\left(a_{1} b-3 b^{2} / 2+1 / 2\right)}\left[\gamma\left(b a_{1}\right)\right]^{-1 / 2} \\
& \times\left[\gamma\left(b a_{1}-b^{2}\right) \gamma\left(a_{1} b+b^{2} / 2+1 / 2\right) \gamma\left(a_{1} b-b^{2} / 2+1 / 2\right)\right]^{-1 / 2} \\
& \times \frac{\gamma\left(1 / 2+b a_{1+3-2} / 2\right) \gamma\left(b a_{1+3-2} / 2-b^{2} / 2\right)}{\gamma\left(1-b a_{1+2+3} / 2+b^{2} / 2\right) \gamma\left(3 / 2-b a_{1+2+3} / 2+b^{2}\right)} \\
& \times \frac{\gamma\left(1 / 2+b a_{1+2-3} / 2\right) \gamma\left(b a_{1+2-3} / 2-b^{2} / 2\right)}{\gamma\left(1 / 2+b a_{2+3-1} / 2\right) \gamma\left(b a_{2+3-1} / 2-b^{2} / 2\right)} .
\end{aligned}
$$

We note that the dependence on the gamma functions containing the different combinations of $a_{i}$ in (127) and (131) exactly cancel, and we obtain (122).

\section{References}

[1] A.Polyakov. Quantum geometry of fermionic strings. Phys.Lett., B103 (1981) 211-213.

[2] F.David. Conformal Field theories coupled to 2-D gravity in the conformal gauge. Mod.Phys.Lett., A3 (1988) 1651.

[3] J.Distler and H.Kawai. Conformal Field theory and 2-D quantum gravity or who's afraid of Joseph Liouville? Nucl.Phys., B231 (1989) 509.

[4] P.Di Francesco, P.Ginsparg and J.Zinn-Justin. 2D Gravity and Random Matrices. Phys.Rep. 254 (1995) 1-133.

[5] A.Belavin, A.Polyakov and A.Zamolodchikov. Infinite conformal symmetry in twodimensional quantum field theory. Nucl.Phys., B241 (1984) 333-380.

[6] T.Curtright, G.Ghandour. Weak Coupling Analysis of the Supersymmetric Liouville Theory. Phys.Lett., B136 (1984) 50.

[7] J.Arvis.Spectrum Of The Supersymmetric Liouville Theory. Nucl.Phys., B212 (1983) 151; ibid B218 (1983) 309.

[8] E.D'Hoker.Classical And Quantal Supersymmetric Liouville Theory. Phys. Rev., D28 (1983) 1346.

[9] O.Babelon. Monodromy Matrix And Its Poisson Brackets In Supersymmetric Liouville String Theory. Phys.Lett., 141B (1984) 353. 
[10] A. Belavin, V. Belavin, A. Neveu, Al. Zamolodchikov. Bootstrap in Supersymmetric Liouville Field Theory. I. NS Sector Nucl.Phys.B784:202-233,2007. e-Print: arXiv:hepth/0703084

[11] V.A. Belavin. On the $\mathrm{N}=1$ super Liouville four-point functions. Nucl.Phys.B798:423442,2008. e-Print: arXiv:0705.1983 [hep-th]

[12] L.Hadasz, Z.Jaskolski, P.Suchanek. Elliptic recurrence representation of the N=1 NeveuSchwarz blocks. Nucl.Phys.B798:363-378,2008. e-Print: arXiv:0711.1619 [hep-th]

[13] L. Hadasz, Z. Jaskolski, P. Suchanek, "Elliptic recurrence representation of the N=1 superconformal blocks in the Ramond sector," arXiv:0810.1203 [hep-th]

[14] V.Kac. Infinite-dimensional Lie algebras. Prog. Math., Vol.44, Birkhäuser, Boston, 1984.

[15] R.Poghossian. Structure Constants in the N=1 Super-Liouville Field Theory. Nucl.Phys. B496 (1997) 451.

[16] R.Rashkov and M.Stanishkov. Three point correlation functions in N=1 super Liouville theory. Phys.Lett., B380 (1996) 49.

[17] Fukuda and K.Hosomichi. Super-Liouville theory with boundary. Nucl. Phys. B635 (2002) 215-254; hep-th/0202032.

[18] H.Dorn and H.-J.Otto. On correlation functions for non-critical strings with $c<1$ but $d>1$. Phys.Lett., B291 (1992) 39, hep-th/9206053;

Two and three point functions in Liouville theory. Nucl.Phys., B429 (1994) 375, hepth/9403141.

[19] A.Zamolodchikov and Al.Zamolodchikov. Conformal bootstrap in Liouville field theory. Nucl.Phys. B477 (1996) 577-605.

[20] A.Belavin, Al.Zamolodchikov. Higher equations of motion in Super Liouville field theory. JETP Lett.84:418-424,2006.

[21] Joseph Polchinski. String theory. Vol. 2: Superstring theory and beyond. Cambridge, UK: Univ. Pr. (1998)

[22] E.Verlinde, H.Verlinde. Lectures On String Perturbation Theory. Published in Trieste School 1988: Superstrings:189

[23] D.Friedan. A Tentative theory of large distance physics. JHEP 0310:063,2003, hep-th/0204131

[24] I.Klebanov and A.Polyakov. Interaction of discrete states in two-dimensional string theory. Mod.Phys.Lett., A6 (1991) 3273-3281, hep-th/9109032. 
[25] E.Witten. Ground ring of two dimensional string theory. Nucl.Phys., B373 (1992) 187, hep-th/9108004.

[26] B.Feigin, private communication.

[27] L.Hadasz, Z. Jaskolski, P.Suchanek. Recursion representation of the Neveu-Schwarz superconformal block. JHEP 0703:032,2007, hep-th/0611266

[28] V.Belavin. N=1 SUSY Conformal Block Recursive Relations. Theor. Math. Phys., 152:1275-1285,2007, hep-th/0611295

[29] A.Belavin and Al.Zamolodchikov. Integrals over moduli spaces, ground ring, and fourpoint function in minimal Liouville gravity. Theor.Math.Phys.147:729-754,2006, hep-th/0510214

[30] A.Belavin and Al.Zamolodchikov. Moduli integrals and ground ring in minimal Liouville gravity. JETP Lett., 82 (2005) 8-14. 